\title{
Estimating the age of Calliphora vicina eggs (Diptera: Calliphoridae): determination of embryonic morphological landmarks and preservation of egg samples
}

\author{
Daniel Martín-Vega ${ }^{1} \cdot$ Martin J. R. Hall $^{1}$
}

Received: 9 October 2015 / Accepted: 15 December 2015 / Published online: 11 January 2016

(C) The Author(s) 2016. This article is published with open access at Springerlink.com

\begin{abstract}
Blow fly eggs may sometimes be the only entomological evidence recovered in a forensic case, especially in cooler weather when hatching might take several days: hence, a method for estimating their age is greatly needed. However, developmental data on blow fly eggs are mainly limited to records of the time to larval hatching. The current paper describes the morphological changes occurring during embryogenesis of the blow fly Calliphora vicina Robineau-Desvoidy and their timing in relation to temperature, in order to determine those characters which can be used for simple egg age estimation using light microscopy. At 7.3 and $25^{\circ} \mathrm{C}, 15$ easily visualised morphological landmarks were determined in C. vicina living embryos, allowing for their age estimation with a resolution of $10-20 \%$ of total egg developmental time. The observed age intervals were compared to the embryonic stages described for the fruit fly Drosophila melanogaster Meigen, which are used as reference data in multiple developmental studies. Moreover, current guidelines for preservation of egg samples, which recommend the placement of living eggs directly into $80 \%$ ethanol, were tested against the hot water killing (HWK) method prior to preservation in $80 \%$ ethanol, recommended for larval and pupal specimens. Direct placement of eggs into $80 \%$ ethanol caused marked decomposition of samples, and no morphological landmarks were discernible. On the other hand, HWK fixation prior to preservation in $80 \%$ ethanol enabled visualisation of 11 of the 15 age-specific morphological landmarks that were discernible in living embryos. Therefore, HWK fixation prior to
\end{abstract}

Daniel Martín-Vega

d.martin-vega@nhm.ac.uk

1 Natural History Museum, Cromwell Road, SW7 5BD London, UK preservation in $80 \%$ ethanol is recommended for egg samples, thus unifying the protocols for collecting entomological evidence.

Keywords Age estimation - Embryonic development . Forensic entomology $\cdot$ Morphology $\cdot$ Post-mortem interval

\section{Introduction}

Throughout its wide geographical distribution, the blow fly Calliphora vicina Robineau-Desvoidy (Diptera: Calliphoridae) is often the first insect colonising cadavers, both indoors and outdoors, frequently promptly after death [1]. Colonisation starts when a female lays her eggs on the cadaver; the offspring then pass through three developmental stages (egg, larva and pupa) before the adult emerges. As the developmental rates of those immature stages are strongly influenced by temperature, a minimum post-mortem interval $\left(\mathrm{PMI}_{\min }\right)$ can be accurately estimated on the basis of forensic scene temperature information and available development data for the pertinent insect species and developmental stage [2]. Quantitative measures of age (e.g. body length) can be modelled in relation to time for C. vicina larvae [3], whereas different approaches have been recently developed for the pupal stage (e.g. [4-6]). However, available development data on blow fly eggs are almost entirely limited to records of the time to larval hatching [7-13], thus restricting the temporal resolution of the egg stage. The egg is by far the shortest developmental stage in blow flies, lasting about $5 \%$ of the total duration of development [7, 10]; hence, the first batch of eggs has usually hatched by the time a cadaver is discovered. Nonetheless, blow fly eggs can sometimes be the only available entomological evidence, especially in cooler weather when hatching might take several days and when, therefore, 
reliable methods for estimating their age become crucial. The use of gene expression profiles is a promising and potential tool for this aim, but it requires further development, considerable expertise and specific fixation and preservation of egg samples [14]. A simple and reliable method for aging blow fly eggs is therefore essential.

A potential approach for estimating the age of blow fly eggs is the use of embryonic morphological landmarks. Developmental studies on the fruit fly Drosophila melanogaster Meigen (Diptera: Drosophilidae) have shown a predictable sequence of morphological changes occurring during embryogenesis [15-18]. Morphological markers have also been used for staging embryos of some Diptera species used in pest control $[19,20]$. Hence, if those morphological landmarks and their timing are determined in the embryonic development of $C$. vicina, they could be used as a qualitative measure of the eggs' age. However, published studies on blow fly embryogenesis focus mainly either on particular stages of the embryonic development $[21,22]$ or on the embryology of particular organs and structures [23-25]. The most comprehensive study to date is that of Starre-van der Molen [26] but, while it describes the internal changes occurring at a cellular level in detail, the external features are only briefly summarized. Moreover, Starre-van der Molen [26] reared C. vicina eggs at room temperature (approximately $22^{\circ} \mathrm{C}$ ), so no accurate data on the timing of the embryonic stages in relation to temperatures can be extracted from those observations.

Furthermore, it is always important to highlight that the analysis of entomological evidence in forensic investigations requires appropriate preserved samples in order to avoid their degradation and other post-mortem changes. In the case of larval specimens, the protocol developed by the European Association for Forensic Entomology (EAFE) recommends the killing and fixation by immersion in hot water (hot water killing or HWK) prior to storage in $80 \%$ ethanol [27], as this method minimizes post-mortem changes in larval length and avoids the marked decomposition of tissues caused by direct placement in ethanol [28]. HWK fixation prior to storage in $80 \%$ ethanol has been shown to be also the best preservation method for pupal specimens [29]. However, for egg specimens, the EAFE protocol [27] recommends killing and preservation by placing the live samples directly into $70-95 \%$ ethanol. Blow fly eggs are protected by the chorion, a resistant and semi-opaque shell which must be removed for effectively visualising the embryo [16-18]. Besides being highly resistant, the chorion shows two dorsal hatching pleats holding a continuous film of air, enabling respiration when the egg is immersed in liquid [30], so the embryo might also continue its development during a certain period of time when placed alive into ethanol. Therefore, a revision of the EAFE guidelines for preservation of egg samples [27] seems necessary.

The current paper describes the morphological changes occurring during the embryonic development of $C$. vicina and their timing, in order to determine those morphological landmarks which can be easily visualised and used for estimating the age of blow fly eggs. Moreover, the quality of preservation of eggs placed directly in $80 \%$ ethanol and eggs HWK fixed prior to storage in $80 \%$ ethanol is tested with the aim of providing a suitable protocol for collecting and preserving egg samples recovered during forensic investigations.

\section{Methods}

A laboratory colony of $C$. vicina was established from adults collected using a modified Redtop ${ }^{\circledR}$ fly trap (Miller Methods, Pretoria) in the Wildlife Garden of the Natural History Museum, London. Newly emerged adults from the colonies were provided with sugar, milk powder and water ad libitum during 1 week, and then also with $2 \mathrm{ml}$ of pig blood (from pig liver) once daily during the following 2 weeks. The flies were then starved for 4-5 days, in order to permit adequate time for egg development and to facilitate predictable egg-laying [31]. After this, fresh pig liver was provided as an oviposition medium for $30 \mathrm{~min}$, and then it was removed and a new piece of fresh pig liver was placed for another $30 \mathrm{~min}$. Only this second batch of eggs was used in the experiment to minimize the occurrence of precocious eggs, which are occasionally matured within the female and laid as larvae [31].

Eggs were collected with a fine brush within $30 \mathrm{~min}$ of oviposition, placed on moistened tissue paper on a Petri dish, and then into an incubator under a constant temperature. The paper was checked at each egg collection and kept moistened by adding water drops; humidity levels in the Petri dish were not measured. Two constant temperatures (temperature \pm standard deviation: $7.3 \pm 0.021{ }^{\circ} \mathrm{C}$ and $25 \pm 0.078{ }^{\circ} \mathrm{C}$ ) were used in this experiment; $7.3{ }^{\circ} \mathrm{C}$ was chosen as a cool temperature under which the embryonic development lasts for several days, while $25{ }^{\circ} \mathrm{C}$ was used for comparison with previous studies on the embryonic development of D. melanogaster [15-18]. Based on data from an unpublished study carried out at the Natural History Museum (Richards et al., unpublished data), the time required from oviposition to hatching by $C$. vicina eggs is approximately $120 \mathrm{~h}$ at $7.3^{\circ} \mathrm{C}$ and approximately $16 \mathrm{~h}$ at $25^{\circ} \mathrm{C}$. Accordingly, 10 eggs were randomly collected at each of the $1110 \%$ time intervals (i.e. from $0 \%$ corresponding to oviposition to $100 \%$ corresponding to hatching), placed in a 1:1 solution of sodium hypochlorite for $5 \mathrm{~min}$, and then rinsed in distilled water for 2-3 min. This method removes the opaque chorion and enables the visualisation of the living embryo through the transparent vitelline membrane [16]. The dechorionated eggs were then placed on a cavity slide in Hoyer's medium and observed and photographed with transmitted light during the following 30 min under a Leica ${ }^{\circledR}$ M165 stereo microscope. The experimental set-up was replicated three times for each temperature, using a different incubator each time to avoid potential instrument bias. 
Resulting images were imported into Adobe Photoshop CS4 (Adobe Systems, Inc.) and adjusted for brightness and contrast in order to improve the display of some internal structures. Terminology follows Campos-Ortega and Hartenstein [17], which includes full definitions for every term. Accumulated degree hours $(\mathrm{ADH})$ required from oviposition to each time interval were calculated for both experimental temperatures using a lower developmental threshold of $1{ }^{\circ} \mathrm{C}$, as determined for C. vicina in London, UK [3].

To test the killing and preservation method, 20 eggs were randomly collected at each $10 \%$ time interval during the $7.3{ }^{\circ} \mathrm{C}$ experimental set-up; the procedure being replicated three times. Ten of those eggs were placed directly into $80 \%$ ethanol and stored at $4{ }^{\circ} \mathrm{C}$, while the other ten eggs were killed by immersion in near-boiling water for $30 \mathrm{~s}$, transferred to $80 \%$ ethanol and stored at $4{ }^{\circ} \mathrm{C}$ for $7-11$ days. After storage, the eggs were dechorionated and visualised following the aforementioned method, and compared with the correspondent living embryos at each time interval.

\section{Results}

\section{Embryonic morphological landmarks}

Fifteen morphological landmarks easily visualised under the stereo microscope were identified in living embryos of C. vicina, allowing for their age estimation with a resolution of $10-20 \%$ of the egg development interval. The chronology of these morphological landmarks is shown in Table 1, including the $\mathrm{ADH}$ required from oviposition to each time interval for both experimental temperatures. The difference in the ADH requirement between both experimental temperatures increased linearly throughout embryogenesis (Fig. 1). The main morphological changes occurring at each $10 \%$ time interval are described below. The correspondence of these intervals to the embryonic stages described for D. melanogaster by Campos-Ortega and Hartenstein [17] is highlighted, as their study includes thorough descriptions of the embryonic events based on histological material and the nomenclature of those stages is typically used in other developmental studies [e.g. 32]. It must be emphasised that the embryonic stages described by Campos-Ortega and Hartenstein [17] are morphological stages, each of them lasting for different time periods.

\section{$0 \%$ (newly laid eggs)}

When oviposited, the egg has just been fertilized, so the zygote fills up the egg giving a uniformly coloured appearance (although bright at the periphery, often brightest at the poles) as the granular yolk mass is homogeneously distributed (Fig. 2a). This homogeneous yolk mass is observable only for a few minutes until the start of cleavage divisions. Indeed, more than $25 \%$ of the specimens showed already the characteristic clear gaps which are diagnostic for the first $10 \%$ interval of embryonic development (Table 1 ). The $0 \%$ interval corresponds to stage 1 of Campos-Ortega and Hartenstein [17].

\section{$10 \%$ of development}

Cleavage divisions take place during this interval of development. There is a contraction of the embryo which leaves two characteristic empty spaces or clear gaps at both anterior and posterior poles (Fig. 2b). The $10 \%$ interval corresponds to stage 2 of Campos-Ortega and Hartenstein [17].

\section{$20 \%$ of development}

The nuclei resultant from the cleavage divisions migrate to the periphery of the embryo and cell membranes extend inwards, so a 'bright peripheral ring' of individual cells is visible around the surface of the embryo (Fig. 2c). The anterior empty space disappears, whereas the posterior space is occupied by the pole cells. Most specimens from the $25^{\circ} \mathrm{C}$ set-up showed this characteristic bright peripheral ring (Table 1), but a relatively low percentage of individuals $(<25 \%)$ showed already an early gastrulation characterised by an irregular surface and bright peripheral ring partially visible (Fig. 2d); in even fewer cases $(<7 \%)$, the bright peripheral ring had completely disappeared, with the cephalic furrow typical of the next development interval partially formed (Fig. 2e). Interestingly, the opposite pattern was found in the $7.3{ }^{\circ} \mathrm{C}$ set-up (Table 1): most specimens showed already an early gastrulation (Fig. 2e), whereas few still showed the bright peripheral ring (Fig. 2c). The $20 \%$ interval corresponds to stages 3 to part of 5 of Campos-Ortega and Hartenstein [17], also including part of stage 6 in those individuals showing an early gastrulation.

\section{$30 \%$ of development}

Gastrulation takes place, and the embryo shows dramatic morphological changes due to endodermal and mesodermal invaginations which form 5-7 divergent dorsal folds (Fig. 2f, g). The formation of the cephalic furrow (Fig. 2f-h) from shortening of lateral cells is also characteristic of this development interval. Dorsal folds and the cephalic furrow were observed in every specimen from this collection interval (Table 1). A clear posterodorsal invagination of the posterior midgut rudiment (Fig. 2f) can be observed in some specimens, but this invagination moves forward and folds into the embryo, disappearing rapidly (Fig. 2g). The $30 \%$ interval corresponds to stages 6 to 9 of Campos-Ortega and Hartenstein [17]. 


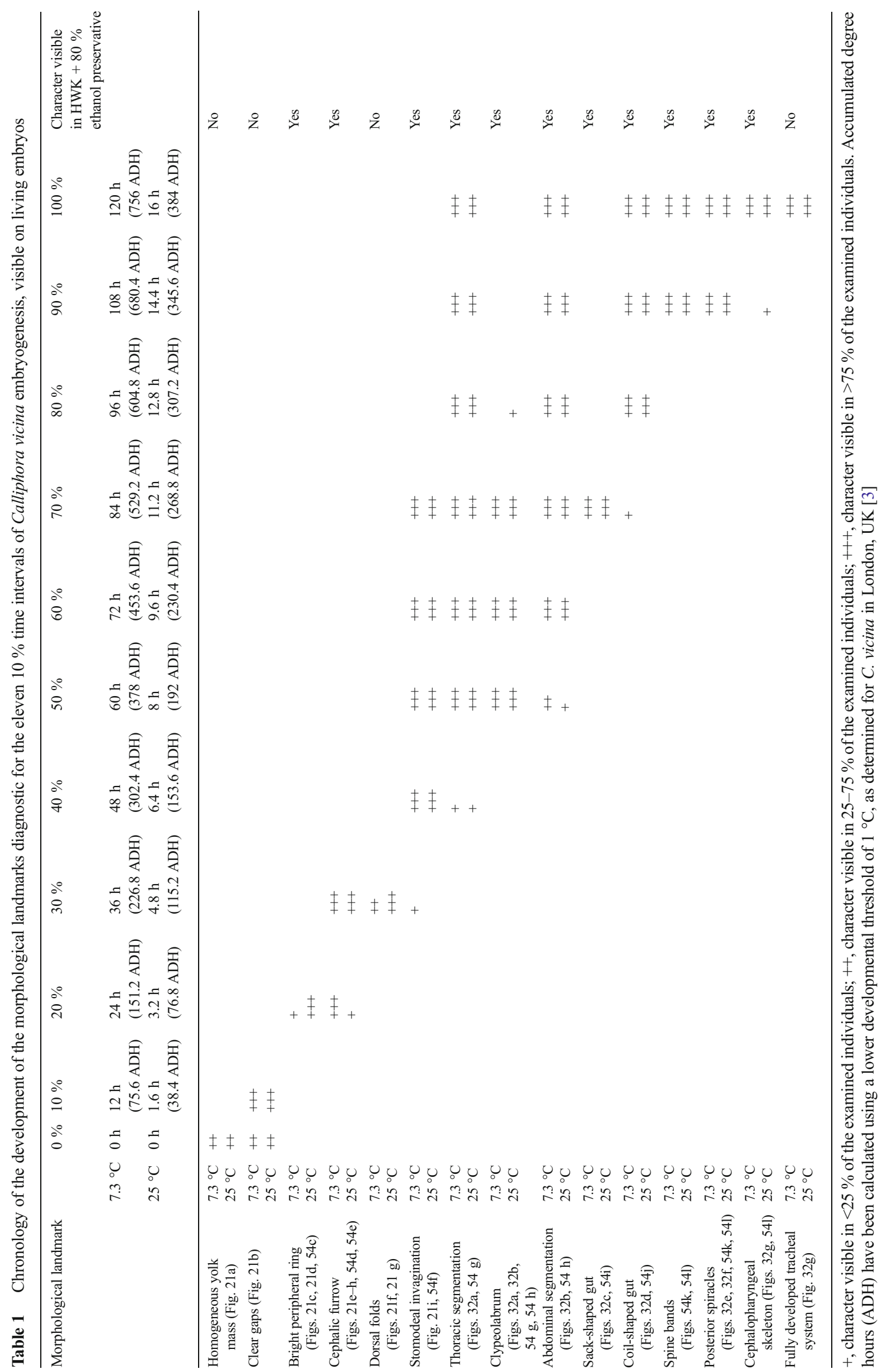




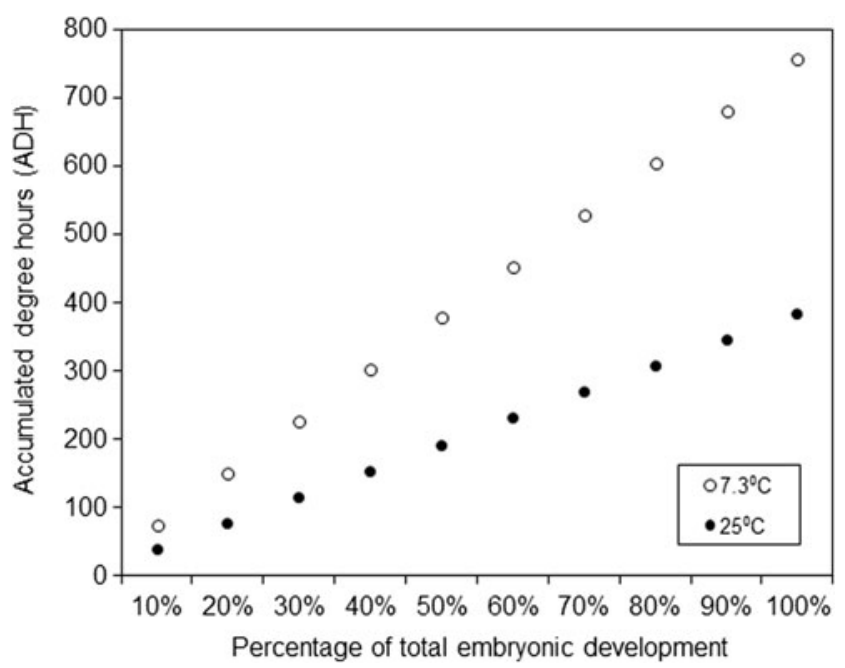

Fig. 1 Accumulated degree hours (ADH) required from oviposition to each time interval at both experimental temperatures. $\mathrm{ADH}$ have been calculated using a lower developmental threshold of $1{ }^{\circ} \mathrm{C}$, as determined for C. vicina in London, UK [3]

\section{$40 \%$ of development}

This developmental interval is characterised by the opening of the stomodeum (i.e. the primordia of the foregut) as a clear, anteroventral invagination of the head segment (Fig. 2i). The germ band, defined as the metameric region of the embryo [17], fully extends around the embryo; an indentation can be clearly observed beside the dorsal tip of the germ band in some specimens (Fig. 2i). Thoracic segmentation is discernible on the ventral surface of some specimens (Table 1). The $40 \%$ interval corresponds to stages 10 and 11 of CamposOrtega and Hartenstein [17].

\section{$50 \%$ of development}

The stomodeum invaginates further and the clypeolabrum (i.e. the fused plate of the clypeus and labrum) can be observed as a clear, anterior protuberance (Fig. 3a). The germ band starts to shorten during this interval and the posterior part of the embryo retracts from the vitelline membrane, leaving a clear gap which is, however, short lived, disappearing by the 60-70\% developmental stage (Fig. 3a). Thoracic segments become distinct on the ventral surface of the embryo; they were observed in every specimen from this and subsequent collection intervals (Table 1). Abdominal segments were also discernible on the ventral surface of a few specimens (Table 1). The $50 \%$ interval corresponds to stage 12 of Campos-Ortega and Hartenstein [17]. a

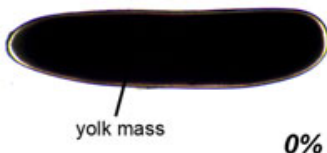

$0 \%$
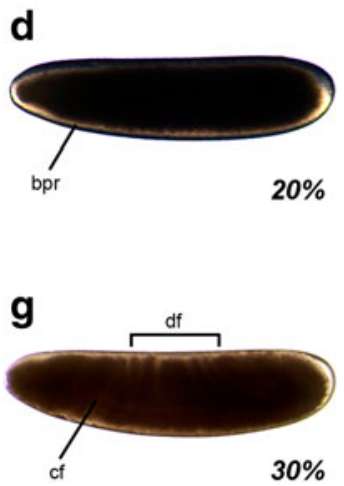

b

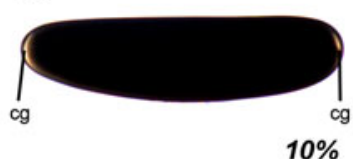

$10 \%$

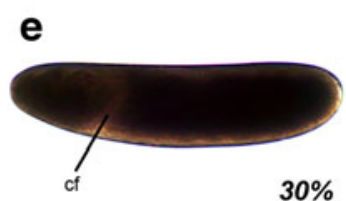

h

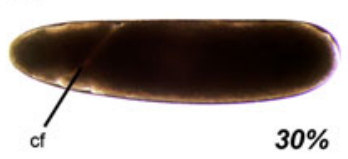

C
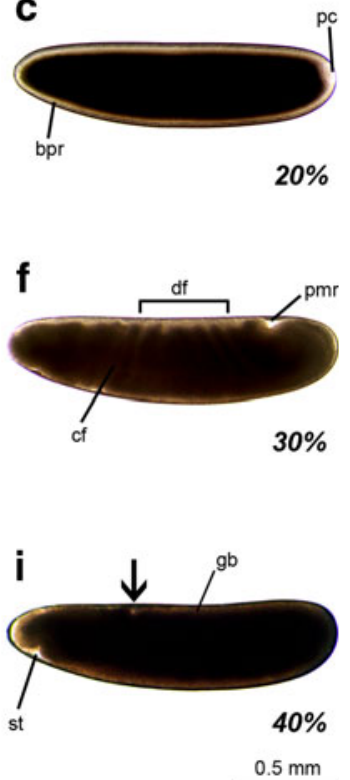

Fig. 2 Embryonic development of Calliphora vicina RobineauDesvoidy at $10 \%$ temporal resolution, $0-40 \%$ time intervals. The corresponding time interval is indicated under each specimen. All the specimens are laterally oriented (anterior part to the left) unless otherwise indicated (h). a $0 \%$ time interval, b $10 \%$ time interval, c $20 \%$ time interval, showing the 'bright peripheral ring' formed by individual cells, d $20 \%$ time interval, specimen showing an early gastrulation but the 'bright peripheral ring' is still discernible, e $20 \%$ time interval, specimen showing an early gastrulation with incomplete formation of the cephalic furrow, f $30 \%$ time interval, specimen showing the posterodorsal invagination of the posterior midgut rudiment, g $30 \%$ time interval, specimen in which the posterodorsal invagination of the posterior midgut rudiment has already disappeared, h $30 \%$ time interval, specimen in ventrolateral view showing the cephalic furrow and i $40 \%$ time interval; the arrow indicates the dorsal indentation beside the tip of the extended germ band. The germ band can be seen as a brighter layer extending over ventral and dorsal sides. $b p r$ 'bright peripheral ring', $c f$ cephalic furrow, $c g$ clear gaps, $d f$ dorsal folds, $g b$ germ band, $p c$ polar cells, $p m r$ invagination of the posterior midgut rudiment, st stomodeal invagination 


\section{$60 \%$ of development}

As the involution of the head segment begins, a dorsal fold starts to progressively cover the clypeolabrum, although it is still clearly distinct (Fig. 3b). The germ band shortens on the dorsal surface and the posterior gap between the embryo and the vitelline membrane almost completely disappears (Fig. 3b). Abdominal segments become evident on the ventral surface of the embryo. The $60 \%$ interval corresponds to stage 13 of Campos-Ortega and Hartenstein [17].

\section{$70 \%$ of development}

The dorsal fold slides over the clypeolabrum and covers it completely (Fig. 3c). In the central region, the gut can be visualised as a closed, 'sack-shaped' tube containing the yolk (Fig. 3c). The $70 \%$ interval corresponds to stages 14 and 15 of Campos-Ortega and Hartenstein [17].

\section{$80 \%$ of development}

The gut becomes 'coil-shaped' as the yolk is gradually digested (Fig. 3d). The gut will be observed until the end of the embryonic development (Table 1), becoming thinner during subsequent development intervals (Fig. 3e). The embryo starts to move actively during this interval. The $80 \%$ interval corresponds to part of stage 16 of Campos-Ortega and Hartenstein [17].

\section{$90 \%$ of development}

Secretion of the cuticle starts at the epidermis and at the tracheal system, so the spine bands and the paired posterior spiracles are discernible (Fig. 3e). However, peristaltic contractions of the gut can be observed and the active movements of the embryo increase significantly, making it difficult to image the spine bands. As the tracheal trunks are progressively filled with air, they can be partially visible in some specimens (Fig. 3f). The sclerotized cephalopharyngeal skeleton can also be discernible in some specimens (Table 1 ). The $90 \%$ interval corresponds to part of stage 16 of Campos-Ortega and Hartenstein [17].

\section{$100 \%$ of development}

The paired tracheal trunks are progressively filled with air and the complete tracheal system is visible (Fig. 3g). The cephalopharyngeal skeleton also becomes clearly distinct. The embryo becomes the first instar larva, which actively tears the vitelline membrane with the tips of the mouth hooks in order to hatch (Fig. 3h). The $100 \%$ interval corresponds to stage 17 of Campos-Ortega and Hartenstein [17].

\section{Preservation of egg samples}

Preservation of eggs in $80 \%$ ethanol without previous fixation resulted in a marked decomposition of tissues, making it impossible to visualise the morphological characters diagnostic for age on the specimens collected from 0 to $80 \%$ timecollection intervals (Fig. 4). Moreover, after several days of storage in $80 \%$ ethanol, the chorion became semi-transparent (Fig. 4d). On the other hand, all the specimens collected at both 90 and $100 \%$ time intervals and placed directly in $80 \%$ ethanol showed the morphology of the first instar larva (Fig. 4d), with some of them having hatched in ethanol (Fig. 4e). In all cases, the larvae showed signs of decomposition and discolouration (Fig. 4d, e).

On the other hand, HWK fixation prior to storage in $80 \%$ ethanol allowed for the visualisation of 11 of the 15 morphological landmarks on preserved eggs (Fig. 5; Table 1), although with less resolution than the living embryos (Figs. 2, 3). As mentioned, the chorion became semi-transparent after several days of storage in $80 \%$ ethanol, sometimes making it possible to identify the morphological landmarks without removing it (Fig. 5h, 1). Collapse of the vitelline membrane prevented the visualisation of the clear gaps typical of the $10 \%$ developmental interval (Fig. 5b, compare with Fig. 2b), as well as the correct visualisation of the dorsal folds characteristic of the $30 \%$ development interval (compare Fig. 5e with Fig. 2f, g), although the cephalic furrow could be observed (Fig. 5e). Moreover, due to protein coagulation by HWK fixation, the tracheal system is not discernible in the $100 \%$ developmental interval (Table 1), while other morphological landmarks (i.e. the posterior spiracles and the cephalopharyngeal skeleton) were sometimes barely discernable (Fig. 51).

\section{Discussion}

\section{Embryonic morphological landmarks}

Previous studies on the egg development of forensically important blow flies suggested that the only external agediagnostic markers in non-dechorionated eggs become visible in the very last hours of the embryonic development, when distinct mouthparts and spine bands can be visualised as the first instar larva is almost ready to hatch [10, 12]. Particularly, Sanit et al. [12] only observed clear spine bands from the 80 to $90 \%$ period of total embryonic development in the blow fly Hypopygiopsis tumrasvini Kurahashi, with no obvious features at earlier stages. This severely limited the temporal resolution of an egg aging method based on the identification of morphological landmarks. However, visualisation of the diagnostic characters can be solved with dechorionation of eggs, as it reveals the morphology of the embryo, making it possible 

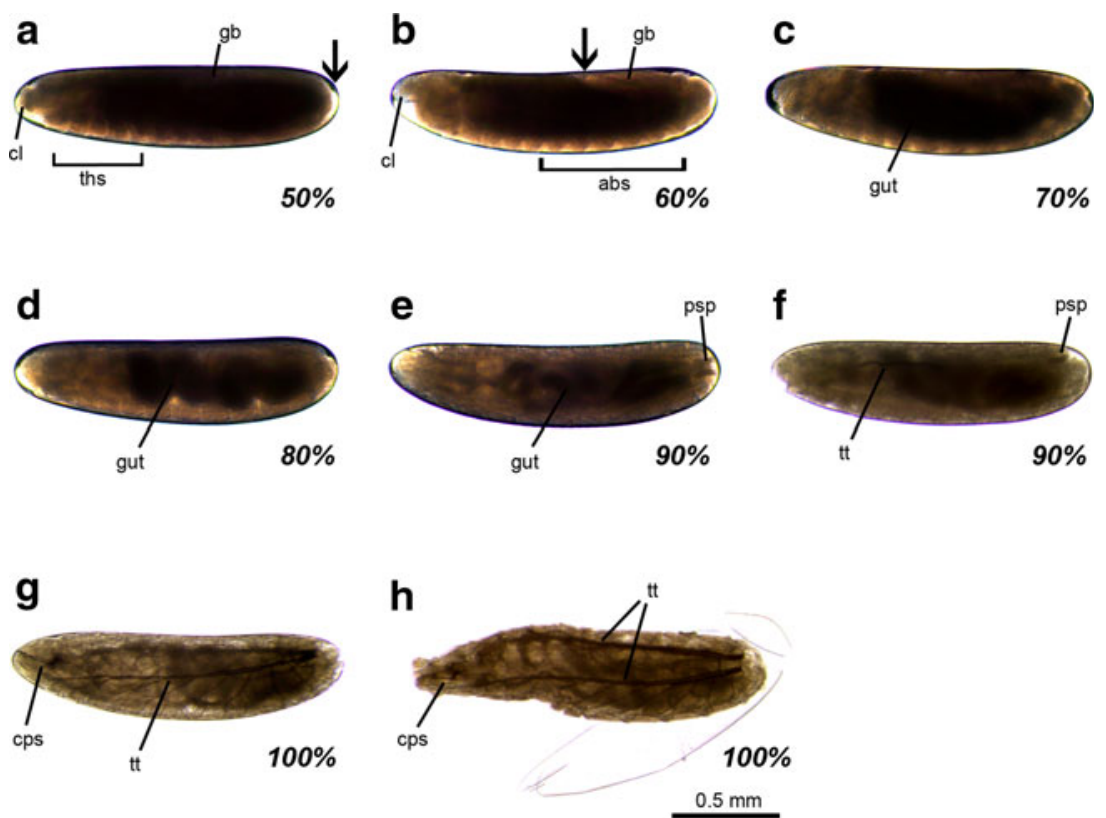

Fig. 3 Embryonic development of Calliphora vicina RobineauDesvoidy at $10 \%$ temporal resolution, $50-100 \%$ time intervals. The corresponding time interval is indicated under each specimen. All the specimens are laterally oriented (anterior part to the left) unless otherwise indicated $(\mathbf{e}, \mathbf{h})$. a $50 \%$ time interval; the arrow indicates the posterior gap left by the contraction of the embryo. The germ band is usually obscured and hardly discernible during this interval. b $60 \%$ time interval; the arrow indicates the dorsal tip of the shortened germ band. c
$70 \%$ time interval, specimen showing 'sack-shaped' gut. d $80 \%$ time interval, specimen showing 'coil-shaped gut'. e $90 \%$ time interval, specimen in dorsal view. f $90 \%$ time interval, specimen with tracheal trunks partially visible. g $100 \%$ time interval. h $100 \%$ time interval, first instar larva in dorsal view hatching from the vitelline membrane. abs abdominal segmentation, $c l$ clypeolabrum, $c p s$ cephalopharyngeal skeleton, $g b$ germ band, $p s p$ posterior spiracles, ths thoracic segmentation, $t t$ tracheal trunk to identify the morphological changes occurring during egg development as already applied to other Diptera species [16, $17,19,20]$. Dechorionation of eggs is a very simple method, not requiring special equipment or particular expertise. Although staining methods can enhance the contrast between cell layers and yolk, thus enhancing the visualisation of some internal morphological characters [18], a simple and fast mounting of the embryos using Hoyer's medium or a solution of sodium chloride [16] is suitable for visualising and identifying the age-specific morphological landmarks determined here (Table 1). It must be noted that the variation in the extent of the germ band during embryonic development (Figs. 2i, 3b) could be an additional informative marker (see CamposOrtega and Hartenstein [17] for further information on this structure). Nevertheless, it has not been included in the current table of diagnostic landmarks (Table 1) because it is not always easily discernible, becoming only prominent during shortening (Fig. 3b), probably due to an increasing cell density [17]. Before shortening, the maximum extent of the germ band can be identified by a dorsal indentation (Fig. 2i), but it must be emphasised that this is a variable feature and frequently obscured by the folds of the serous membrane [17].

Overall, the morphological characters described and their chronology are consistent with those described for other Diptera species [15-20,32], including the previous study on
C. vicina embryogenesis [26]. Nevertheless, it must be highlighted that the 5-7 divergent dorsal folds observed during gastrulation at $30 \%$ of embryonic development of $C$. vicina (Fig. 2f, g) are more numerous than the three folds observed in D. melanogaster [17], being more similar to the up to six folds observed in the more closely related blow fly Lucilia sericata (Meigen) [32]. Mellethin et al. [32] suggested that such higher number of dorsal folds formed during gastrulation might be due to the different dimensions of the body axes, with a much greater length-to-thickness ratio in blow fly eggs than in Drosophila eggs.

Regarding the timing of the developmental intervals, it must be noted that previous descriptions of the embryonic stages included only approximations of the duration of each stage and highlighted the difficulty of delimiting the embryonic stages [17]. Indeed, when applying the current agespecific morphological landmarks (Table 1), it should be taken into account that embryonic development is a continuous process and that the morphological events observed at the end of a development interval continue during part of the following interval. In any case, the time intervals described here are consistent with the times suggested for $C$. vicina embryogenesis by Starre-van der Molen [26]. Interestingly though, when compared to the times suggested for D. melanogaster at $25^{\circ} \mathrm{C}$ [17], the first stages of embryonic development appear to 
a

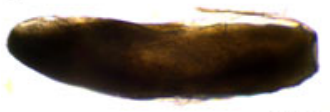

$20 \%$ b

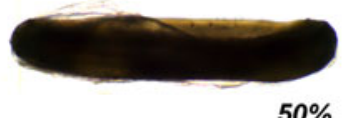

C

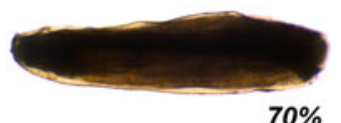

d

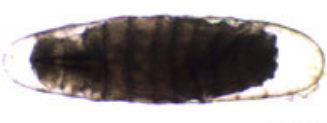

$90 \%$

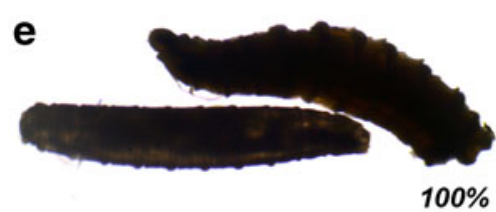

$0.5 \mathrm{~mm}$
Fig. 4 Eggs of Calliphora vicina Robineau-Desvoidy collected at $10 \%$ time intervals of the total embryonic development and placed directly in $80 \%$ ethanol. The corresponding time interval is indicated under each specimen. All the specimens are laterally oriented (anterior part to the left) unless otherwise indicated (d). a $20 \%$ time interval, b $50 \%$ time interval,

progress slightly faster in C. vicina (Table 1). Mellenthin et al. [32] also suggested shorter stages in the first part of the c $70 \%$ time interval and d $90 \%$ time interval, specimen in ventral view showing the anatomy of a first instar larva. Note that the chorion has not been removed. e $100 \%$ time interval, first instar larvae hatched during storage in $80 \%$ ethanol

embryonic development of $L$. sericata. Different duration of the embryonic stages has been observed in closely related a

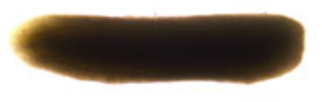

$0 \%$

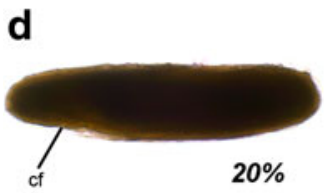

g
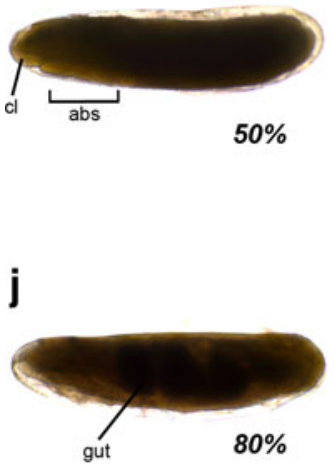

b

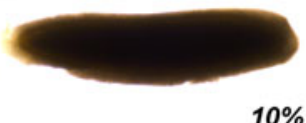

C

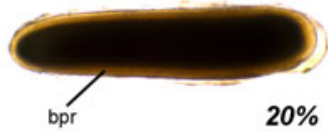

f

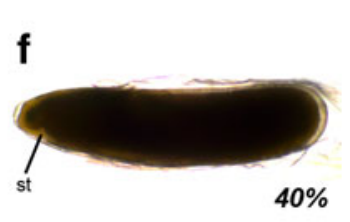

i

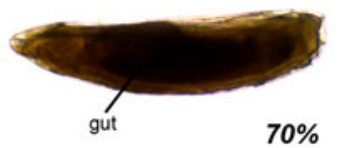

$20 \%$ h

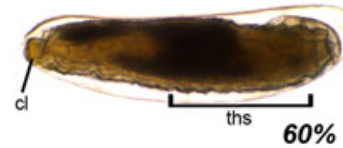

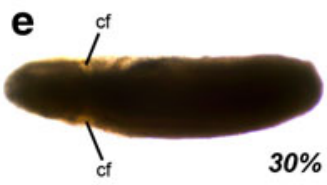
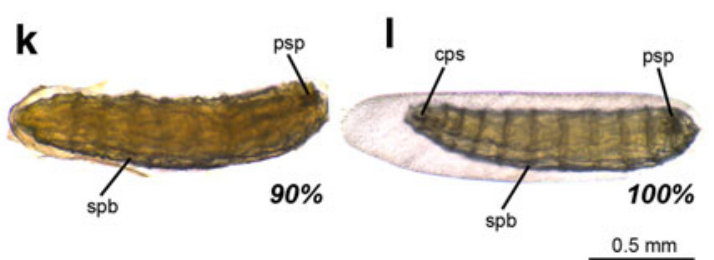

Fig. 5 Eggs of Calliphora vicina Robineau-Desvoidy collected at different $10 \%$ time intervals of the total embryonic development and preserved in $80 \%$ ethanol for 1-2 weeks after hot water killing (HWK) fixation. The corresponding time interval is indicated under each specimen. All the specimens are laterally oriented (anterior part to the left) unless otherwise indicated (e). a $0 \%$ time interval, b $10 \%$ time interval, c $20 \%$ time interval, showing the 'bright peripheral ring' formed by individual cells, d $20 \%$ time interval, specimen showing an early gastrulation with incomplete formation of the cephalic furrow, $\mathbf{e}$
$30 \%$ time interval, specimen in ventral view showing the cephalic furrow, f $40 \%$ time interval, g $50 \%$ time interval and h $60 \%$ time interval. Note that the chorion has not been removed. i $70 \%$ time interval, specimen showing 'sack-shaped' gut, j $80 \%$ time interval, specimen showing 'coil-shaped gut', k $90 \%$ time interval and $\mathbf{l} 100 \%$ time interval. Note that the chorion has not been removed. abs abdominal segmentation, $b p r$ 'bright peripheral ring', $c f$ cephalic furrow, $c l$ clypeolabrum, $c p s$ cephalopharyngeal skeleton, $p s p$ posterior spiracles, $s p b$ spine bands, st stomodeal invagination, ths thoracic segmentation 
species of the genus Drosophila [20], so the development intervals determined in the current study for $C$. vicina might not be valid for other Calliphoridae species.

It is worth mentioning the difference observed at the $20 \%$ development interval between the two temperature set-ups (Table 1), suggesting a proportionately slightly shorter development during the first stages of embryogenesis at lower temperatures. Lower tolerance of young embryos to cold has been observed in L. sericata [33] and Drosophila suzukii (Matsumura) [20], so shortening of earlier stages at lower temperatures might be an adaptive strategy of cold-tolerant species like $C$. vicina. However, our current data do not allow us to draw further conclusions on this issue and, to the best of our knowledge, there are no studies on the duration of the embryonic stages at different temperatures in other blow fly species. It is also beyond the scope of the current study to discuss the difference in the $\mathrm{ADH}$ requirements between the two experimental temperatures (Fig. 1). However, our result concurs with other studies of $C$. vicina that report higher values of $\mathrm{ADH}$ at lower temperatures [3, 27, 34]. This variation requires further investigation, including use of more experimental temperatures than the two used in the present study.

\section{Preservation of egg samples}

Direct placement of living entomological samples into ethanol has been shown to cause marked decomposition of tissues following death of the samples, frequently resulting in shrinkage and discolouration $[4,28,29]$; this is also clearly the case in blow fly eggs as indicated by the current results (Fig. 4). Moreover, as ethanol does not instantly kill the specimens, it might lead to erroneous $\mathrm{PMI}_{\min }$ estimations, particularly in the last hours of egg development, when first instar larvae may hatch in the preservative (Fig. 4). For these reasons, direct placement of live eggs into ethanol should be avoided when collecting egg samples at the forensic scene.

As mentioned, HWK fixation prior to preservation in $80 \%$ ethanol is already recommended for larval and pupal samples collected at forensic scenes [27, 29]. This fixation and preservation method also allows for the visualisation of most agediagnostic morphological landmarks in egg samples (Fig. 5; Table 1); hence, it is also recommended for eggs. Dechorionation may not be strictly needed in some cases as the chorion usually becomes semi-transparent after several days of storage and the fixed embryo can be visualised inside (Figs. 5h, 1). However, to achieve the best possible resolution, it is preferable in most cases to remove the chorion.

Finally, as a guideline for forensic practice, the egg samples collected at a forensic scene should ideally be divided into two batches: one should be placed on moistened tissue paper in vials, stored in a cool bag with a temperature data logger and transferred to an expert for rearing as soon as possible, and the other one should be HWK fixed (as soon as possible after collection, recording the time) prior to storage in $80 \%$ ethanol for a subsequent morphological analysis at the laboratory. This recommendation of fixation and storage method unifies the protocol for collecting entomological evidence as already recommended for both larval and pupal samples [27, 29].

Acknowledgments We are grateful to Matteo Mondani for helping to stimulate this study. Two anonymous reviewers provided constructive comments and suggestions which improved the current manuscript. The first author is supported by an EC funded Marie Curie Intra-European Fellowship (FP7-PEOPLE-2013-IEF n: 624575).

\section{Compliance with ethical standards}

Funding This study was supported by an EC funded Marie Curie IntraEuropean Fellowship (FP7-PEOPLE-2013-IEF n: 624575).

Conflict of interest The authors declare that they have no competing interest.

Open Access This article is distributed under the terms of the Creative Commons Attribution 4.0 International License (http:// creativecommons.org/licenses/by/4.0/), which permits unrestricted use, distribution, and reproduction in any medium, provided you give appropriate credit to the original author(s) and the source, provide a link to the Creative Commons license, and indicate if changes were made.

\section{References}

1. Reibe S, Madea B (2010) How promptly do blowflies colonise fresh carcasses? A study comparing indoor with outdoor locations. Forensic Sci Int 195:52-57. doi:10.1016/j.forsciint.2009.11.009

2. Amendt J, Richards CS, Campobasso CP, Zehner R, Hall MJR (2011) Forensic entomology: applications and limitations. Forensic Sci Med Pathol 7:379-392. doi:10.1007/s12024-0109209-2

3. Donovan SE, Hall MJR, Turner BD, Moncrieff CB (2006) Larval growth rates of the blowfly, Calliphora vicina, over a range of temperatures. Med Vet Entomol 20:106-114. doi:10.1111/j.13652915.2006.00600.x

4. Richards CS, Simonsen TJ, Abel RL, Hall MJR, Schwyn DA, Wicklein M (2012) Virtual forensic entomology: improving estimates of minimum post-mortem interval with 3D micro-computed tomography. Forensic Sci Int 220:251-264. doi:10.1016/j.forsciint. 2012.03.012

5. Brown K, Thorne A, Harvey M (2015) Calliphora vicina (Diptera: Calliphoridae) pupae: a timeline of external morphological development and a new age and PMI estimation tool. Int J Legal Med 129:835-850. doi:10.1007/s00414-014-1068-Z

6. Zajac BK, Amendt J, Horres R, Verhoff MA, Zehner R (2015) De novo transcriptome analysis and highly sensitive digital gene expression profiling of Calliphora vicina (Diptera: Calliphoridae) pupae using MACE (Massive Analysis of cDNA Ends). Forensic Sci Int Genet 15:137-146. doi:10.1016/j.fsigen.2014.11.013

7. Anderson GS (2000) Minimum and maximum development rates of some forensically important Calliphoridae (Diptera). J Forensic Sci 45:824-832 
8. Anderson GS (2004) Determining time of death using blow fly eggs in the early postmortem interval. Int J Legal Med 118:240-241. doi: 10.1007/s00414-004-0443-6

9. VanLaerhoven SL, Anderson GS (2001) Implications of using development rates of blow fly (Diptera: Calliphoridae) eggs to determine post-mortem interval. J Entomol Soc Brit Columbia 98:189194

10. Greenberg B, Kunich JC (2002) Entomology and the law. Cambridge University Press, Cambridge

11. Bourel B, Callet B, Hédouin V, Gosset D (2003) Flies eggs: a new method for the estimation of short-term post-mortem interval? Forensic Sci Int 135:27-34. doi:10.1016/s0379-0738(03)00157-9

12. Sanit S, Sukontason K, Klong-klaew T, Tomberlin JK, Limsopatham K, Samerjai C, Sontigun N, Sukontason KL (2014) Ontogenesis and developmental rate of the blow fly, Hypopygiopsis tumrasvini Kurahashi (Diptera: Calliphoridae). Trop Biomed 31:760-768

13. Alonso MA, Souza CM, Linhares AX, Thyssen PJ (2015) Egg developmental time and survival of Chrysomya megacephala and Chrysomya putoria (Diptera: Calliphoridae) under different temperatures. J Med Entomol 52:551-556. doi:10.1093/jme/tjv066

14. Tarone AM, Jennings KC, Foran DR (2007) Aging blow fly eggs using gene expression: a feasibility study. J Forensic Sci 52:1350 1354. doi:10.1111/j.1556-4029.2007.00587.x

15. Imaizumi $T$ (1958) Recherches sur l'expression des facteurs létaux héréditaires chez l' embryon de la drosophile V. Sur l'embryogénèse et le mode des létalités au cours du développement embryonnaire. Cytologia 23:270-285. doi:10. 1508/cytologia.23.270

16. Bownes M (1975) A photographic study of development in the living embryo of Drosophila melanogaster. J Embryol Exp Morph 33:789-801

17. Campos-Ortega JA, Hartenstein V (1985) The embryonic development of Drosophila melanogaster. Springer, Berlin. doi:10.1007/ 978-3-662-02454-6

18. Wieschaus E, Nüsslein-Volhard C (1998) Looking at the embryos. In: Roberts DB (ed) Drosophila: A practical approach, 2nd edn. Oxford University Press, Oxford, pp 179-214

19. Havelka J, Landa V Jr, Landa V (2007) Embryogenesis of Aphidoletes aphidimyza (Diptera: Cecidomyiidae): morphological markers for staging living embryos. Eur J Entomol 104:81-87. doi: 10.14411/eje.2007.013

20. Landi S, Gargani E, Paoli F, Simoni S, Roversi PF (2015) Morphological markers for cryopreservation in the embryonic development of Drosophila suzukii (Diptera: Drosophilidae). J Econ Entomol, in press. doi: 10.1093/jee/tov114
21. Lowne BT (1892) The anatomy, physiology, morphology and development of the blowfly, vol I. RH Porter, London. doi:10.5962/ bhl.title.66964

22. Strasburger EH (1934) Über die Zellbewegungen bei der Eifurchung der Fliege Calliphora erythrocephala Meigen. Z Wiss Zool 145:625-641

23. Henson H (1944) The embryology and metamorphosis of the alimentary canal in the blowfly (Calliphora). Proc Leeds Phil Lit Soc (Sci Sect) 4:184-190

24. Ludwig CE (1949) Embryology and morphology of the larval head of Calliphora erythrocephala (Meigen). Microentomology 14:75111

25. Schoeller J (1964) Recherches descriptives et expérimentales sur la céphalogenèse de Calliphora erythrocephala (Meigen), au cours des développements embryonnaire et postembryonnaire. Arch Zool Exp Gén 103:1-216

26. van der Starre-van der Molen LG (1972) Embryogenesis of Calliphora erythrocephala Meigen. I. Morphology. Neth J Zool 22:119-182. doi:10.1163/002829672x00059

27. Amendt J, Campobasso CP, Gaudry E, Reiter C, LeBlanc HN, Hall MJR (2007) Best practice in forensic entomology-standards and guidelines. Int J Legal Med 121:90-104. doi:10.1007/s00414-0060086-x

28. Adams ZJO, Hall MJR (2003) Methods used for the killing and preservation of blowfly larvae, and their effect on post-mortem larval length. Forensic Sci Int 138:50-61. doi:10.1016/j.forsciint. 2003.08.010

29. Brown K, Thorne A, Harvey M (2012) Preservation of Calliphora vicina (Diptera: Calliphoridae) pupae for use in post-mortem interval estimation. Forensic Sci Int 223:176-183. doi:10.1016/j. forsciint.2012.08.029

30. Hinton HE (1960) The chorionic plastron and its role in the eggs of the Muscinae (Diptera). Quart J Micr Sci 101:313-332

31. Davies K, Harvey M (2012) Precocious egg development in the blowfly Calliphora vicina: implications for developmental studies and post-mortem interval estimation. Med Vet Entomol 26:300 306. doi:10.1111/j.1365-2915.2011.01004.x

32. Mellenthin K, Fahmy K, Ali RA, Hunding A, Da Rocha S, Baumgartner S (2006) Wingless signaling in a large insect, the blowfly Lucilia sericata: a beautiful example of evolutionary developmental biology. Dev Dynam 235:347-360. doi:10.1002/ dvdy.20632

33. Zhang B, Numata H, Mitsui H, Goto SG (2008) Short-term cold storage of blowfly Lucilia sericata embryos. Insect Sci 15:225228. doi:10.1111/j.1744-7917.2008.00204.x

34. Greenberg B (1991) Flies as forensic indicators. J Med Entomol 28: 565-577, http://dx.doi.org/10.1093/jmedent/28.5.565 